\title{
PRECIOS AGROPECUARIOS E INGRESOS: EL CASO DE TRES PROVINCIAS EN ECUADOR
}

\author{
Manuel Chiriboga ${ }^{*}$, Edwin Vasquez ${ }^{*}$ y Octavio Sotomayor ${ }^{*}$
}

\section{Introducción}

La situación del sector agrícola en el mundo entero ha enfrentado cambios importantes en los tres últimos años, al experimentar un incremento sustantivo en los precios de los alimentos, en términos nominales, superiores a sus picos más altos de los años 1974-1975, 1980-1981 y 19951996. Son varios los factores que han contribuido a ese escalonamiento histórico, entre ellos tenemos el aumento en el uso de bienes agrícolas como fuentes energéticas alternativas (biocombustibles); la asociación entre el comportamiento de los precios del petróleo y precios agrícolas; el incremento en la demanda de algunos países emergentes entre los cuales se destacan China e India, que han sufrido importantes tasas de crecimiento en sus economías; los efectos del cambio climático sobre la producción de alimentos que han reducido sus niveles de oferta y; factores de carácter especulativo por parte de inversionistas de corto plazo.

Estos cambios en el mercado agrícola traen consigo implicaciones sobre las economías mundiales, en especial, sobre aquellos países en que la agricultura es una de las principales fuentes generadoras de ingreso y empleo, como es el caso del Ecuador; al igual que en países en donde la dependencia en el consumo de alimentos de origen importado es alto. Frente a esta coyuntura surgió la necesidad de evaluar cuál fue el efecto real de los altos precios de los bienes agrícolas sobre el ingreso real de los pequeños productores, identificar si este escenario se constituyó en una oportunidad favorable para mejorar sus condiciones de vida y específicamente el acceso a mayores y mejores alimentos.

* Investigador de Rimisp y director del Observatorio de Comercio Exterior del Ecuador; Vásquez y Sotomayor son investigadores de este último. El trabajo se realizó con el auspicio del Instituto Interamericano de Cooperación para la Agricultura (IICA) y fue revisado y editado por Julio Paz Cafferata, consultor del IICA. 
La investigación se concentró en tres provincias del Ecuador: El Oro, Los Ríos y Chimborazo, seleccionadas por la importancia que en ellas tienen los pequeños y medianos productores agrícolas. La población sujeta del estudio fue un grupo de pequeños productores.

Para la medición de los impactos sobre los agricultores del Ecuador, se utilizó una metodología basada en la cuantificación de las variaciones en el ingreso de los factores de producción en el sector agrícola, para lo cual fue necesario visualizar los cambios experimentados tanto en los ingresos brutos o valores de venta de la producción resultante por hectárea en cada uno de los productos seleccionados, como también de los costos de producción por hectárea, en especial del componente de insumos materiales directos que también se vieron afectados por la evolución de sus precios internos. Lo que se pretende evaluar es el cambio en el valor agregado por hectárea, motivado por el cambio de precios en los productos e insumos de la agricultura. Por lo anterior, los jornales y los gastos en servicios (extensión, fumigación, sanidad, transporte y otros similares) quedan como parte del valor agregado por la producción agrícola y que se distribuyen a los factores de producción que la hicieron posible (sala- rios a la mano de obra, honorarios a los servicios, rentas de la tierra, intereses financieros, utilidades del capital).

La medición cuantitativa se realizó para el periodo 2000-2008, poniendo énfasis, más que a los valores absolutos, al comportamiento de los índices para medir los cambios reales presentados en el periodo de análisis. Se consideró como año base el 2005, desde el que comienzan a observase las variaciones de precios. La información obtenida se basó en datos del III Censo Nacional Agropecuario, las encuestas anuales de cultivos del INEC hasta el 2007, previsiones de productores y expertos para el 2008 dado la ausencia de datos en el último año y de información del sistema de precios del Ministerio de Agricultura, Ganadería y Pesca.

Como parte del trabajo se efectuó un levantamiento de información de campo en un grupo de entre 35 y 40 productores en cada una de las provincias en estudio, con la finalidad de obtener la percepción de los productores agrarios acerca del impacto que ha tenido el aumento de los precios de los productos básicos y de las materia primas en temas relacionados con sus ingresos y su seguridad alimentaria, así como medir la percepción de las acciones o medidas que han tenido que adoptar en res- 
puesta al aumento de los precios de los productos básicos y de las materias primas.

\section{Los precios agrícolas inter- nacionales y la seguridad ali- mentaria en Ecuador}

\subsection{Comportamiento de los precios agrícolas internacionales}

El mercado de productos agrícolas históricamente ha presentado continuas fluctuaciones y volatilidades en sus precios. De manera permanente se han observado ciclos de costos altos y bajos, sino obstante, desde el 2005, pero de manera más pronunciada en el año 2006, se entra en una etapa de incremento considerable de los precios. Las cotizaciones de algunos de los grupos de productos como cereales, lácteos y aceites alcanzaron los niveles de importes más altos en la historia. Sin embargo, en el segundo semestre del 2008 todos los costos de los productos básicos en el mercado internacional, incluidos los agrícolas, empezaron a descender de los picos alcanzados, algunos productos lo hicieron antes. No obstante lo anterior, los precios promedio para productos agrícolas en el 2008, aún se mantienen por encima de sus correspondientes al 2005.
Según la información de precios del Fondo Monetario Internacional (FMI) los costos de los alimentos crecieron en un $80 \%$ entre el 2005 y junio del 2008, para luego decrecer en $-33 \%$ en el segundo semestre. El crecimiento en los distintos grupos de productos alimenticios tuvo un ritmo diferente, en función a los desarrollos de las respectivas demandas y ofertas, así como por la situación de partida de los correspondientes niveles de inventario en el mundo entero. Entre ellos los de mayor incremento son los cereales, cuyos montos llegaron en el segundo trimestre del 2008 a ser $161 \%$ más caros que su promedio en el 2005, y el grupo de los aceites que aumentaron en un $130 \%$ en el mismo periodo. Ambos grupos tuvieron caídas de precios superiores a $-40 \%$ en el segundo semestre del 2008. El incremento de los costos de los productos lácteos fue muy acelerado entre el 2005 y el 2007 (108\%), pero éstos empezaron a caer al final de dicho año. Los valores de los productos cárnicos y de los productos tropicales aumentaron menos que el promedio de los alimentos, en especial los primeros, que además fueron afectados por el fuerte aumento en los costos de sus insumos (granos forrajeros). La información más 
reciente indica una ligera recuperación de precios en enero de 2009, en todos los productos alimenticios, salvo los productos lácteos ${ }^{1}$.

Cuadro $\mathrm{N}^{\circ} 1$

Mercado internacional: Variaciones de precios de productos alimenticios Porcentajes

\begin{tabular}{|l|c|c|c|c|}
\hline $\begin{array}{l}\text { Grupos de } \\
\text { Productos }\end{array}$ & $\begin{array}{c}\text { Máximo } \\
\text { Precio }\end{array}$ & $\begin{array}{c}\text { Max. precio/ } \\
\text { Prom. 2005 }\end{array}$ & $\begin{array}{c}\text { Dic. 2008/ } \\
\text { Max. precio }\end{array}$ & $\begin{array}{c}\text { Ene. 2009/ } \\
\text { Dic. 2008 }\end{array}$ \\
\hline Alimentos & Jun 08 & $80,0 \%$ & $-33,4 \%$ & $6,4 \%$ \\
Cereales & Abr-08' & $161,0 \%$ & $-40,4 \%$ & $9,5 \%$ \\
Aceites & Jun-08 & $130,0 \%$ & $-42,9 \%$ & $10,0 \%$ \\
Cárnicos & Ago-08' & $18,0 \%$ & $-20,2 \%$ & $2,0 \%$ \\
Lácteos & Nov-07' & $108,0 \%$ & $-43,0 \%$ & $-14,0 \%$ \\
Tropicales & Jul-08' & $56,0 \%$ & $-20,7 \%$ & $5,0 \%$ \\
\hline
\end{tabular}

Fuente: IICA con datos IMF

Este comportamiento de los precios tiene su explicación en la confluencia de factores tanto de oferta como de demanda que se han presentado en la economía mundial en los últimos años y que ya los mencionamos anteriormente. Otro factor que influyó en la espiral alcista de precios fueron las operaciones especulativas en los mercados financieros de productos agrícolas básicos, que permitieron diversificar las carteras y reducir el riesgo de las bolsas de valores. También ha contribuido para esto la aplicación de algunas políticas públicas orientadas a frenar el efecto inflacionario de la escala de precios tales como prohibiciones o aumentos de impuestos a la exportación, las que han terminado exacerbando más la volatilidad de los precios.

Este comportamiento del mercado de productos agrícolas en una época de globalización económica, caracterizada por la apertura de mercados y las reducciones de algunas políticas de protección en el marco de normas multilaterales que rige el comercio de alimentos, presenta una trasmisión más rápida sobre las economías domésticas. De ahí que en el 2008 se ha presentado un aumento 
en los niveles de inflación de las economías mundiales, afectando los niveles de ingreso de la población.

Si bien los efectos sobre los precios de los bienes alimenticios de la canasta de consumo han sido casi inmediatos, es importante determinar si se presenta una transmisión efectiva hacia el ingreso de los productores, lo cual dependerá de las estructuras de los mercados y de los diferentes factores que limitan o favorecen esa transferencia de precios.

Analizando los niveles de correlación de los precios internacionales con los del productor y consumidor para el caso del Ecuador, se observa que dependiendo del tipo de producto, la mayor relación se presenta a nivel de los precios al consumidor que a nivel del productor.

Cuadro $\mathrm{N}^{\circ} 2$

Niveles de correlación de los precios internacionales

Periodo 2000-2008

\begin{tabular}{|l|c|c|}
\hline Productos & Int./productor & Int./consumo \\
\hline Arroz & 0,77 & 0,91 \\
Soya- Aceite vegetal & 0,63 & 0,96 \\
Leche & 0,63 & 0,67 \\
Maíz duro - Pollo & 0,94 & 0,73 \\
Trigo - Pan & - & 0,85 \\
Cacao & 0,97 & - \\
Café & 0,95 & - \\
Banano & 0,54 & - \\
Cebada & 0,80 & 0,94 \\
\hline
\end{tabular}

Fuente: SG CAN, FAO, Magap

Elaboración: OCE

Los niveles de trasmisión de precios en el caso del Ecuador son diferenciados de acuerdo al tipo de producto. En este aspecto actúan diferentes factores que hacen que en unos productos la transmisión sea más directa que en otros, y tienen que ver con las políticas públicas, tales como controles de precios al consumidor en el caso de la leche y el arroz; políticas de precios de referencia de importación y absorción de cosecha en el caso del maíz duro y soya; políticas de precios oficiales al productor en el caso del banano; las políticas arancelarias y el uso de permisos de importación, entre otros.

También tiene implicación la forma cómo los productos se articulan a los diferentes mercados de 
exportación, agroindustriales o de consumo, y la presencia de agentes intermediarios que mediante los márgenes de comercialización van restando los beneficios entre las diferentes cadenas de comercialización, como son los casos de leche, arroz, soya, cebada y el propio banano.

En definitiva, hay una nueva realidad que se está viviendo en los mercados agrícolas a partir del 2006, y en mayor grado, desde el 2008; sin embargo, así como se experimentaron de una manera rápida incrementos sustanciales de los precios de los bienes agrícolas a la fecha de este estudio (primer trimestre 2009), la tendencia comienza a declinar como resultado de la crisis financiera mundial surgida en Estados Unidos por la explosión de la burbuja inmobiliaria, lo que hace difícil prever la evolución futura de los mercados agrícolas.

\subsection{Análisis de la seguridad alimen- taria en Ecuador}

El comportamiento experimentado por los mercados agrícolas en los últimos años, ha puesto en debate su relación con los posibles efectos hacia la seguridad alimentaria, considerando dos de sus principales aspectos: 1. El riesgo respecto a la disponibilidad de alimentos, y 2. El riesgo respecto al acceso a los ali- mentos por todos los sectores de la población. El perfil del Ecuador en relación a estos dos elementos nos permite apreciar los niveles de vulnerabilidad y potencialidad para hacer frente a la nueva realidad de los mercados agrícolas y los posibles cambios que experimentará la economía mundial como resultado de la crisis financiera internacional.

\subsubsection{Nivel de autosuficiencia alimen- taria del Ecuador}

El mayor riesgo en la disponibilidad de alimentos se asocia generalmente con el grado de abastecimiento interno de los productos de la dieta alimenticia nacional. A mayor porcentaje de abastecimiento interno se considera que menor es el riesgo de escasez alimentaria a nivel del país.

En el caso del Ecuador, considerando los siete grupos de alimentos esenciales en la dieta calórica de la población, sólo en aceites vegetales y cereales las importaciones tienen un peso importante sobre la oferta de alimentos. El peso de las importaciones en la presente década ha crecido al pasar en el caso de los aceites vegetales del $26 \%$ en el 2000 al $40 \%$ en el 2007; mientras que en el caso de los cereales es del $24 \%$ en el 2000 al 33\% en el 2007. En los otros grupos de productos: carnes, frutas, leches, 
vegetales y raíces la dependencia en el abastecimiento de alimentos de producción nacional es bastante alta, cubriendo la demanda entre un $95 \%$ a cerca del $100 \%$.

En el caso de los grupos de cereales y aceites vegetales, vale mencionar que el mayor peso de las importaciones se presenta en cuatro productos que en los últimos diez años han reducido su producción local: trigo, cebada, maíz y aceite de soya, que aportan con $3 \%, 6 \%, 50 \%$ y $35 \%$ respectivamente. El poco peso de las importaciones alimenticias se explica por varios factores, pero con seguridad el más importante ha sido la tradicional protección arancelaria y permisos de importación, a los que han sido sujetos la mayor parte de productos alimenticios. A pesar de esas políticas, el peso de los alimentos importados durante el periodo 2000-2007, desde el punto de vista del consumo de calorías, ha crecido al pasar del $17 \%$ al $26 \%$ registrando una tasa de crecimiento del $6 \%$ anual. Sin embargo, ese crecimiento se basa en los mismos productos mencionados: trigo, cebada, maíz y aceite de soya.

En algunos casos el incremento de las importaciones de estos productos no necesariamente es el resultado de un debilitamiento o reducción sustantiva de la producción, sino del crecimiento del consumo de determinados productos en la dieta de la población, en especial de bienes procesados que utilizan algunos de estos productos como materia prima, tal es el caso de la cebada y el maíz en la producción de cerveza, pollo o alimentos procesados como hojuelas de maíz (cornflakes), entre otros.

\subsubsection{Financiamiento de la importa- ción de alimentos}

Debido a que el Ecuador tendría que adquirir en el exterior una parte de sus necesidades alimenticias a precios bastante más elevados que los pagados a inicios de la década; existe la preocupación sobre la capacidad del país a nivel agregado para adquirir los faltantes en el mercado internacional. Para evaluar el grado de vulnerabilidad de la economía ante los cambios en el comportamiento de los mercados agrícolas, es recomendable utilizar una serie de indicadores macroeconómicos como la tasa de crecimiento de la economía en su conjunto, el comportamiento del ingreso nacional, la situación de la balanza en cuenta corriente y el nivel de reservas internacionales en relación a los posibles gastos requeridos para la importación de alimentos. 
La economía ecuatoriana ha tenido un alto crecimiento en lo que va de la presente década, con una tasa anual del 5\% de crecimiento promedio de su Producto Interno Bruto (PIB). Sin embargo, dicho crecimiento ha sido irregular a través de los años, destacándose picos con tasas mayores al 5\% en el 2004, 2005 y 2008, y tasas menores en los años anteriores. En términos percápita, los mayores aumentos en el PIB se dieron en el 2004 y 2008.

Un indicador más apropiado para evaluar la capacidad de la economía ecuatoriana en su conjunto para hacer frente a los mayores costos de los alimentos importados es la evolución del Ingreso Nacional Bruto (INB) percápita, que incluye en su cálculo las ganancias o pérdidas de términos de intercambio externo como una forma de medir las variaciones del poder adquisitivo de la producción nacional destinada al mercado externo. En épocas de mejoras en los términos de intercambio el INB tiende a ser mayor que el PIB, debido a que por la mejora relativa de los precios de exportación se obtiene más importaciones por la misma producción exportada. Esto significa un mayor ingreso a partir de la misma producción. En caso de pérdida de términos de intercambio, sucede lo inverso.

Lo anterior puede verse en el Cuadro 3, donde en los años en que suben los índices de términos de intercambio, la tasa de crecimiento anual del PIB percápita resulta inferior a la tasa del INB percápita, y viceversa. Así, en el 2005 la gran ganancia en términos de intercambio externos, se tradujo en un crecimiento record del ingreso percápita del $12,4 \%$, mientras que el PIB percápita aumentó únicamente en un $4,8 \%$. Para el año 2008, si bien aún no se dispone de cifras oficiales del INB, es posible prever un aumento aún mayor que el registrado en el 2005 en el INB percápita, debido a que el PIB percápita tuvo la mayor tasa de aumento de la década y que ésta fue acompañada por una significativa mejora en los términos de intercambio externos.

En resumen, las condiciones de la economía ecuatoriana en términos de crecimiento del producto y de los ingresos durante el periodo 20052008 fueron óptimas para enfrentar el aumento en los precios agrícolas internacionales. 
Precios agropecuarios e ingresos: el caso de tres provincias en Ecuador

Cuadro $\mathrm{N}^{\circ} 3$

Evolución del Producto Interno Bruto e Ingreso Nacional Bruto (percápita, 2000-2008)

\begin{tabular}{|c|c|c|c|c|c|}
\hline Años & $\begin{array}{c}\text { PIB per } \\
\text { cápita } \\
\text { (US\$) }\end{array}$ & $\begin{array}{l}\text { INB per } \\
\text { cápita } \\
\text { (US\$) }\end{array}$ & $\begin{array}{c}\text { Crecimiento } \\
\text { PIB } \\
\text { percápita (\%) }\end{array}$ & $\begin{array}{c}\text { Crecimiento } \\
\text { INB } \\
\text { percápita (\%) }\end{array}$ & $\begin{array}{c}\text { Términos de } \\
\text { intercambio } \\
\text { externos (Px/Pm) }\end{array}$ \\
\hline 2000 & 1.296 & 1.290 & & & 100,0 \\
2001 & 1.347 & 1.306 & $4,0 \%$ & $1,2 \%$ & 90,4 \\
2002 & 1.387 & 1.360 & $3,0 \%$ & $4,2 \%$ & 98,0 \\
2003 & 1.420 & 1.388 & $2,4 \%$ & $2,1 \%$ & 96,6 \\
2004 & 1.516 & 1.473 & $6,8 \%$ & $6,1 \%$ & 105,5 \\
2005 & 1.589 & 1.656 & $4,8 \%$ & $12,4 \%$ & 112,7 \\
2006 & 1.634 & 1.783 & $2,8 \%$ & $7,6 \%$ & 117,0 \\
2007 & 1.648 & 1.828 & $0,9 \%$ & $2,6 \%$ & 129,7 \\
2008 & 1.764 & - & $7,1 \%$ & & \\
\hline
\end{tabular}

Fuente: Banco Central de Ecuador y World Economic Outlook (IMF), Abril 2009

Para visualizar el nivel de vulnerabilidad frente a shocks externos para hacer frente a las necesidades de importación de bienes y servicios, así como con los compromisos de capital con el exterior, requeridos para el normal desenvolvimiento de la economía nacional, lo más relevante es analizar los flujos de financiamiento externo del Ecuador. Las principales variables que determinan un saldo positivo o negativo de la cuenta corriente son el saldo de la balanza comercial (exportaciones menos importaciones) y el balance de transferencias, los cuales a lo largo del periodo han presentado saldos favorables. Los otros componentes como son el balance de servicio y de renta han sido desfavorables durante el periodo de análisis, siendo más bien factores de desahorro de la cuenta corriente del Ecuador.

En los escenarios que se están analizando, de crecimiento de precios de las materias primas y los productos básicos en los mercados internacionales, su efecto sobre algunas de las variables macroeconómicas, entre ellas, la balanza de pagos, ha sido positiva, por cuanto ha permitido que uno de sus principales rubros de financiamiento como son las exportaciones hayan experimentado un importante crecimiento en los últimos cuatro años.

La balanza comercial del Ecuador depende en gran porcentaje de las exportaciones de petróleo y alimentos, entre ellos: banano, café, cacao, 
flores, camarón, etcétera. El conjunto de bienes primarios representa aproximadamente un $80 \%$ de sus exportaciones totales. El aumento de precios del petróleo y de algunos bienes alimenticios han beneficiado el ingreso de divisas, durante el periodo 2000-2007; las exportaciones se han incrementado a una tasa promedio anual del 16\%.

A esto se suma el crecimiento de las divisas procedentes de las remesas del exterior que, en el mismo periodo, aumentaron a una tasa del $10 \%$ del promedio anual, al pasar de 1.351 millones de dólares en el 2001 a 3.354 millones de dólares en el 2007, nivel similar inclusive al de la reserva monetaria del Ecuador.

Sin embargo, desde finales del 2008, la situación de la balanza de pagos se ha vuelto problemática por la reducción de sus dos principales fuentes de financiamiento. Las exportaciones del Ecuador están disminuyendo por la caída sustantiva de los precios del petróleo y se presenta una reducción de las remesas ante la crisis financiera internacional. Ello constituye una peligrosa vulnerabilidad de la economía ante los problemas económicos internacionales $y$, por ende, pone en riesgo las fuentes de financiamiento de la importación de alimentos, en especial de aquellos que tienen una alta dependencia de importaciones.

El grado de cobertura que ofrecen las reservas internacionales del país respecto a los requerimientos de divisas para la adquisición de alimentos es otro indicador usado para medir el grado de riesgo respecto a la suficiente disponibilidad alimentaria. En el caso del Ecuador, el comportamiento de la reserva internacional ha sido positivo a lo largo del periodo 2000-2007, registrando una tasa de crecimiento promedio anual del $20 \%$, alcanzando su mayor crecimiento del 2003 al 2005 y en el 2007. Estos recursos de libre disponibilidad son un activo importante que permitiría cubrir los requerimientos de importaciones y de divisas en caso de presentarse algún desfase o crisis en el balance de cuenta corriente del país.

En el caso extremo que se requiriera utilizar el total de las reservas que mantiene el Banco Central del Ecuador en activos externos para cubrir el monto total de importaciones de alimentos, considerando la información del 2007, se podría cubrir o precautelar aproximadamente unos 36 meses (más de 3 años) de las necesidades de importación. 
2.2.1 Condiciones de acceso a los alimentos en el Ecuador

En América Latina, en general, el problema de seguridad alimentaria es básicamente de personas o familias de bajos ingresos, que impide a ciertos grupos de la población acceder a alimentos en el mercado. Los problemas de disponibilidad son de carácter temporal, provocados muchas veces por fenómenos climáticos y referidos a localidades específicas. Por lo tanto, los indicadores útiles para evaluar los cambios en la capacidad de acceso de la población a los alimentos se orientan a medir los cambios en los ingresos de sus segmentos más pobres.

\subsubsection{Los niveles de pobreza}

Los niveles de pobreza en el Ecuador se han reducido en los últimos tres años, a nivel nacional se pasó del $51,2 \%$ en el 2004, al 43\%, en el 2006. Los índices más altos de pobreza se presentan en la zona rural, y se mantienen cercanos al 50\%.

En cuanto a los grados de indigencia, éstos también han disminuido en los últimos tres años al pasar del $22,3 \%$ en el 2004 , al $16,1 \%$ en el 2006, tendencia que se observa tanto en la zona urbana como en el sector rural. El mayor nivel de indigencia se presenta a nivel rural al llegar en el 2004 al 30\% de la población, si bien para el 2006 se redujo al 22\% sigue manteniéndose el porcentaje más alto frente a la población urbana.

\subsubsection{Los niveles de empleo y salarios}

El crecimiento económico del Ecuador en los últimos seis años, ha permitido una reducción importante del desempleo. Mientras en el $2001 \mathrm{el}$ desempleo a nivel urbano se encontraba en el 10,4\% de la PEA, para el 2005 este se redujo al 8,5\% de la PEA y para el 2007 al 7,4\%, sin embargo de ello, en el 2009 presenta un incremento al $8,3 \%$.

El comportamiento de la economía ecuatoriana durante los últimos años ha favorecido la recuperación del ingreso real de los trabajadores, medido por el comportamiento del salario mínimo real. Para el año 2008 el salario real frente al 2003 ha mejorado en un $26 \%$, siendo su recuperación constante y sistemática ${ }^{2}$.

2 Uno de los factores que ha permitido el mejoramiento de los ingresos reales de los trabajadores ha sido el esquema monetario de dolarización que estabilizó los niveles de precios y favoreció la mejora del nivel adquisitivo. 


\subsubsection{El gasto público en el área social}

Finalmente, durante el periodo 2000-2008 el gasto fiscal o público orientado al sector social por habitante medido a precios corrientes del 2000, también experimentó crecimientos tanto a nivel total como en determinados rubros: educación, salud y seguro social, no así el de la vivienda que se mantuvo estable.

El gasto total por habitante se incrementó de 51 dólares en el 2000 a 118 dólares en el 2008, lo que implicó un crecimiento promedio anual del $14 \%$. El mayor crecimiento del gasto se presentó en el año 2004 con un $18 \%$, manteniéndose el resto del periodo en tasas de entre el $4 \%$ y $5 \%$. El gasto social percápita como proporción al PIB percápita a precios del 2000, se incrementó en el periodo al pasar de un $4 \%$ en el 2000 a un 6\% en el 2008, si bien es una proporción pequeña del ingreso promedio nacional, sí mejoró en términos reales la inversión pública sobre el área social.

De los principales rubros del componente social, durante el 20002008 los mayores gastos públicos se realizaron en las áreas de educación y seguridad social, que juntos representaron más del $70 \%$ del total, mientras que el rubro de menor peso fue el de vivienda representando menos del 5\% del total, no obstante, para el 2008 éste último es uno de los rubros de mayor crecimiento.

La inversión del gasto público en las áreas sociales marca la línea de las políticas hacia la atención de la pobreza y el mejoramiento de los ingresos reales de la población. Durante los últimos ocho años la inversión social no se ha reducido, ha tratado de mantenerse en niveles similares como una proporción del ingreso nacional por habitante, pero es necesario definir acciones para ampliar su cobertura, en especial hacia zonas rurales que es dónde se concentran los mayores niveles de pobreza.

\section{Análisis del impacto del aumento de precios sobre el ingreso neto de los producto- res}

La medición de los efectos del incremento de precios sobre los pequeños productores agrícolas se ha concentrado en el análisis de estudios de caso para tres provincias del Ecuador (El Oro, Los Ríos y Chimborazo), y en los tipos de productos, que tienen un peso importante en su estructura productiva.

El Oro es una de las provincias en que las actividades agrícolas influye 
de manera importante en el desarrollo de su economía. La agricultura es la actividad económica con el mayor peso en la generación del PIB local alcanzando un $19 \%$, seguida por el sector del comercio que llega al 17\%; en tercer lugar tenemos al sector de la construcción que llega al 12\%; posteriormente esta la industria manufacturera con el $9 \%$ y al final están actividades como la pesca y el transporte que alcanzan el $8 \%$.

En la provincia de El Oro, la mayor parte de su superficie agrícola se dedica a cultivos permanentes (alrededor de 84 mil hectáreas) y en menor medida a la producción de cultivos transitorios, pastos, barbechos, montes, entre otros. Las actividades agrícolas se concentran en nueve productos que representan el 93\% de la producción bruta del sector: banano, cacao, café, arroz, maíz duro seco, maíz suave seco y caña de azúcar. Veremos que sólo el banano representa el $83 \%$ del valor total de la producción agrícola de la provincia.

La provincia de Los Ríos depende en una alta proporción de las actividades agrícolas. El PIB agrícola representa el 38\% del total provincial, y es la participación más alta si se compara con el resto de provincias del país. Le siguen en importancia el comercio al por mayor y menor que alcanza el 17\%; seguido del transpor- te con un $10 \%$ y por último las actividades inmobiliarias que llegan al $9 \%$. De hecho buena parte del comercio y el transporte se relacionan con la actividad agropecuaria.

Los Ríos tiene unas $637 \mathrm{mil} \mathrm{hec-}$ táreas que representan el 5\% del territorio a nivel nacional, de las cuales un 34\% (217 mil hectáreas) están destinada a cultivos permanentes; un $8 \%$ a cultivos transitorios; un $28 \%$ a barbecho; el $11 \%$ a siembra de pastos $y$ en menores proporciones a descanso, pastos y montes. A nivel de cultivos permanentes representa el $16 \%$ del total nacional y, en cuanto a cultivos transitorios un $12 \%$ a nivel nacional siendo una de las provincias de mayor participación en el uso del suelo para cultivos.

Las actividades agrícolas en la provincia de Los Ríos se concentran en diez productos que representan el $90 \%$ de la producción bruta del sector: arroz, maíz duro, soya, cacao, banano, plátano, yuca, palma africana, maracuyá y café, de los cuales los cuatro primeros representaron el $86 \%$ de los ingresos totales de la provincia.

En la provincia del Chimborazo, la agricultura constituye la tercera actividad económica en relación a su participación en el PIB provincial $(12 \%)$, detrás del sector comercio con un $23 \%$ y del sector transporte 
que representa el $14 \%$. Chimborazo tiene unas 471 mil hectáreas que representan el $4 \%$ del territorio a nivel nacional. Un $10 \%$ de esta superficie se dedica a cultivos transitorios, el $11 \%$ a siembra de pastos y sólo el 1\% (5.6 mil hectáreas) está destinada a cultivos permanentes. Esta provincia concentra un alto número de pequeños productores (de menos del 10\% de hectáreas), con alrededor del 59\% de productores de esta categoría a nivel nacional.

Las actividades agrícolas en Chimborazo se concentran en ocho productos que representan el $85 \%$ de la producción bruta del sector: leche, cebada, maíz suave seco, papa, haba, maíz suave, choclo y trigo, de las cuales las cuatro primeras representaron el $80 \%$ de los ingresos totales de la provincia. Las actividades en el Chimborazo se caracterizan porque su producción tiene una alta orientación al autoconsumo en especial en el grupo de pequeños productores.

\subsection{Evolución de precios de productos e insumos a nivel del productor}

3.1.1 Evolución de los precios de productos

$\mathrm{Al}$ productor de banano el precio le aumentó sustancialmente en los dos últimos años (2007-08), hecho favorable a la principal producción de la provincia del El Oro, en que muchos pequeños productores se especializan. La fruta se destina en

Cuadro $\mathrm{N}^{\circ} 4$

El Oro: índices de precios al productor (Índices 2005-100)

\begin{tabular}{|l|r|r|r|r|}
\hline \multirow{2}{*}{ Años } & Banano & Cacao & Café & \multirow{2}{*}{$\begin{array}{c}\text { Indice } \\
\text { El Oro }\end{array}$} \\
\cline { 2 - 3 } \multicolumn{3}{|c|}{ Indice } & 24,9 \\
\hline 2000 & 23,1 & 53,6 & & 82,6 \\
2001 & 84,0 & 62,5 & 29,8 & 92,6 \\
2002 & 91,4 & 112,5 & 29,1 & 90,5 \\
2003 & 88,9 & 117,1 & 34,0 & 88,7 \\
2004 & 88,4 & 94,5 & 53,3 & 100,0 \\
2005 & 100,0 & 100,0 & 100,0 & 82,7 \\
2006 & 80,7 & 113,0 & 102,2 & 18,9 \\
2007 & 116,6 & 158,6 & 120,5 & 140,4 \\
2008 & 137,1 & 190,5 & 158,3 & \\
\hline
\end{tabular}

Fuente: MAGAP, INEC, ANECAFE (para café arabigo

Elaboración: OCE 
gran porcentaje hacia mercados emergentes como es el caso de Rusia, la mayoría de productores trabajan bajo la modalidad spot en la venta de la fruta, por ende son más vulnerables a las variaciones de precios que se presentan en dichos mercados.

Las políticas de los exportadores de banano están en función de su mapa de mercados y de la oferta internacional de la fruta, esto hace que muchas veces lo que recibe el exportador no necesariamente se traduce al productor en mejores precios, además de la influencia que tiene la política interna de fijación de precios al productor.

El precio del cacao muestra dos etapas de comportamiento, una antes del 2005 en donde hay una alta variabilidad, y otra posterior en donde se presenta una continua alza en los precios, que llegó en el último año a más del $90 \%$ respecto al año base.

Cuadro $\mathrm{N}^{\circ} 5$

Los Ríos: índices de precios al productor

(Índices 2005-100)

\begin{tabular}{|l|r|r|r|r|c|}
\hline \multirow{2}{*}{ Años } & Arroz & Cacao & Maíz duro & Soya & $\begin{array}{c}\text { Indice } \\
\text { Los Ríos }\end{array}$ \\
\cline { 2 - 4 } & \multicolumn{3}{|c|}{ Indice } \\
\hline 2000 & 65,8 & 53,6 & 78,0 & 56,4 & 65,2 \\
2001 & 57,1 & 62,5 & 77,1 & 83,4 & 64,7 \\
2002 & 55,5 & 112,5 & 91,9 & 79,4 & 77,9 \\
2003 & 63,6 & 117,1 & 80,8 & 78,5 & 80,3 \\
2004 & 103,1 & 94,5 & 96,9 & 78,7 & 98,0 \\
2005 & 100,0 & 100,0 & 100,0 & 100,0 & 100,0 \\
2006 & 71,0 & 113,0 & 99,1 & 100,8 & 88,7 \\
2007 & 99,6 & 158,6 & 119,0 & 105,4 & 117,3 \\
2008 & 263.7 & 190,5 & 142,8 & 182,8 & 214,5 \\
\hline
\end{tabular}

Fuente: MAGAP, INEC

Elaboración: OCE

El precio del arroz recibido por el productor tuvo un crecimiento espectacular en el 2008 al incrementarse en un $164 \%$ frente al periodo de base. Si bien el comportamiento en los precios internacionales tuvo su influencia en esta importante alza, también influyeron las condiciones climáticas de inicios de año, con fuertes inundaciones, lo que afectó 
algunas zonas arroceras, reduciendo la superficie destinada a este cultivo y la oferta del producto.

El maíz amarillo duro, al igual que el arroz, está sujeto a una política arancelaria de estabilización de precios conocida como Sistema Andino de Franja de Precios (SAFP), que determina que el costo de importación del maíz importado es un referente para el precio interno del producto nacional. El Ministerio de Agricultura establece un precio de referencia basado en el costo de importación. Adicionalmente, existe una política por la cual se asegura que la industria se provea primero de la producción local, bajo un mecanismo de absorción de cosecha, antes de autorizarse la importación de maíz.

Si bien la producción de maíz duro tiene una importante participación en el abastecimiento de la demanda interna, no cubre la totalidad de los requerimientos, por lo que la industria requiere importar el déficit de consumo. Esta realidad de la cadena productiva genera una articulación muy directa entre el comportamiento del precio internacional con el precio doméstico, más aún cuando el costo de importación resultante de la aplicación del SAFP se traduce en un precio de referencia que el industrial debe pagar al productor. El maíz duro fue uno de los productos que sufrió las más importantes escaladas de precios a nivel internacional en los últimos años, observándose un comportamiento similar en el precio doméstico, el cual se incrementó en un $19 \%$ en el 2007 y en un $43 \%$ en el 2008 frente al año de base.

El cultivo de la soya al igual que el maíz está articulado al sector agroindustrial, pero su demanda está abastecida por producción interna en sólo un $19 \%$, por lo que los requerimientos de producto importado son muy altos. Esta situación convierte a este cultivo en uno de los más vulnerables frente a las fluctuaciones del mercado internacional y a la competencia del producto importado. Al igual que en el caso del maíz, este producto está sujeto a la aplicación del SAFP y de mecanismos de precios y absorción de cosecha, además de que es un cultivo que se utiliza de manera complementaria al maíz en época de verano como ciclo de rotación de la tierra.

En la provincia del Chimborazo no se presentan, prácticamente, variaciones sustanciales en los precios recibidos por los productores de sus principales productos agropecuarios, si bien se observa ligeros crecimientos en los años 2007 y 2008 no guardan relación con las variaciones presentadas en las cotizaciones internacionales. 
Precios agropecuarios e ingresos: el caso de tres provincias en Ecuador

Cuadro $\mathrm{N}^{\circ} 6$

Chimborazo: índices de precios al productor

(Índices 2005-100)

\begin{tabular}{|c|c|r|r|r|r|}
\hline \multirow{2}{*}{ Años } & Cebada & Papa & Leche & Maíz suave & \multirow{2}{*}{$\begin{array}{c}\text { Indice } \\
\text { Chimborazo }\end{array}$} \\
\cline { 2 - 4 } & \multicolumn{3}{|c|}{ Indice } & 68,5 \\
2000 & 100,3 & 81,4 & 59,6 & 100,5 & 87,8 \\
2001 & 119,0 & 53,9 & 96,3 & 124,2 & 95,4 \\
2002 & 106,6 & 86,4 & 96,7 & 118,3 & 89,4 \\
2004 & 85,3 & 72,5 & 96,5 & 82,1 & 84,6 \\
2005 & 105,1 & 55,9 & 95,0 & 75,0 & 100,0 \\
2006 & 100,0 & 100,0 & 100,0 & 100,0 & 101,4 \\
2007 & 97,8 & 105,1 & 99,6 & 111,4 & 95,2 \\
2008 & 136,1 & 69,7 & 100,3 & 128,1 & 113,5 \\
\hline
\end{tabular}

Fuente: MAGAP, INEC

Elaboración: OCE

La producción de leche, al estar concentrada en pequeños productores, no tiene una articulación muy fuerte con las industrias lácteas y su producción es comercializada por los intermediarios o está destinada a las queserías de la zona. La leche que se dirige a las industrias requiere de ciertas condiciones de infraestructura tales como depósitos de refrigeración que existen en reducida cantidad en la zona, de ahí que una buena parte de la producción de los pequeños productores es vendida a queserías, intermediarios o se destina al autoconsumo, recibiendo menores precios que si lo hicieran directamente a las industrias. Adicional- mente, el nivel de precios de la leche ha estado sujeto a controles de diverso tipo.

La papa, el maíz suave y la cebada son productos que no están vinculados directamente al mercado internacional y sus precios internos están más influenciados por la situación de la oferta y demanda internas. Sin embargo, existe un importante estimulo a su demanda cuando los precios de otros alimentos transables internacionalmente aumentan y los consumidores sustituyen dichos consumos por alimentos de menor precio. Estos precios tienen un incremento importante en 2008, en relación al año base. 
3.1.2 Comportamiento de los costos de insumos materiales directos

Para determinar el efecto de los aumentos de precios sobre el costo en insumos directos se identificó el peso que tiene cada uno de ellos en la estructura de los costos de producción. En el caso de los cultivos se los dividió en semillas, fertilizantes, herbicidas, insecticidas, fungicidas y otros, y para el caso de la leche se adicionaron los rubros de alimentación de ganado, sanidad animal y combustibles y energía.

Cabe anotar que en el análisis del comportamiento de los costos de materiales directos de producción se consideró como supuesto que el volumen de insumo utilizado por hectárea se mantuvo constante en el tiempo y que el nivel y composición de dichos costos sólo varió en función de los precios de los insumos y productos. Este supuesto equivale a considerar que no existió sustitución entre insumos, a pesar de los cambios en sus precios relativos.

En la mayoría de los cultivos los fertilizantes llevan el mayor peso dentro de los insumos transables, con participaciones que llegan hasta el $87 \%$, en el caso del café. El producto con el peso más bajo en uso de fertilizantes es el banano con un $12 \%$, debido a la exigencia que tiene este producto en los controles fitosanitarios, en especial de la sigatoka negra que requiere de procesos permanentes de fumigación para controlar el hongo, justamente el uso de fungicidas en este cultivo es uno de los más altos de todo el grupo con un $21 \%$ de participación en la estructura del costo. Otro rubro importante en los costos son las cajas, bolsas y cartones que se utilizan en el proceso de producción y embalaje de algunos productos como el banano, arroz, maíz y cacao. Estos materiales son generalmente importados por lo que las variaciones de sus precios se han asimilado a las variaciones en los costos de importación de productos intermedios desde los Estados Unidos.

Los otros componentes, salvo algunos casos como el arroz y la soya, en el uso de herbicidas, presentan un peso menor en los costos de producción, por lo que la mayor sensibilidad sobre los costos se presentaría en las variaciones que sufra ya sea a nivel de precios o de abastecimiento de fertilizantes.

En el caso de la leche los principales componentes son los alimentos balanceados para ganado, sin embargo, en gran parte la alimentación del ganado utiliza pastos naturales o cul- 
tivados, en cuyo caso los gastos se presentan en fertilizantes y semillas.

Los mayores cambios en los precios de insumos agrícolas, respecto a los que regían en el año 2005, se presentan en los fertilizantes que duplicaron sus precios para el 2008. En una magnitud cercana aumentaron también las vacunas y medicinas veterinarias $(83 \%)$ y los fungicidas (81\%). El resto de insumos registra aumentos inferiores al 29\%. En el caso de combustibles y energía, la relativa estabilidad del índice refleja la política de subsidio estatal a dichos rubros de costos.

Cuadro $\mathrm{N}^{\circ} 7$

Índice de precios de insumos agrícolas (Índices 2005-100)

\begin{tabular}{|l|c|c|c|c|c|c|c|c|}
\hline Años & Fertilizantes & Herbicidas & Insecticidas & Fungicidas & Otros & $\begin{array}{c}\text { Alimentación } \\
\text { Ganado }\end{array}$ & $\begin{array}{c}\text { Sanidad } \\
\text { Animal }\end{array}$ & $\begin{array}{c}\text { Com. } y \\
\text { Energía }\end{array}$ \\
\hline 2000 & 0,57 & 0,89 & 1,01 & 0,48 & & 1,19 & 1,11 & \\
2001 & 0,47 & 0,96 & 1,10 & 0,44 & 0,84 & 0,53 & 1,11 & 0,63 \\
2002 & 0,48 & 0,94 & 1,01 & 1,00 & 0,83 & 0,70 & 1,15 & 0,72 \\
2003 & 0,62 & 0,79 & 1,02 & 1,11 & 0,87 & 1,27 & 1,14 & 0,98 \\
2004 & 0,85 & 0,87 & 0,90 & 1,06 & 0,92 & 0,96 & 1,09 & 0,99 \\
2005 & 1,00 & 1,00 & 1,00 & 1,00 & 1,00 & 1,00 & 1,00 & 1,00 \\
2006 & 0,98 & 0,87 & 1,02 & 0,93 & 1,07 & 1,05 & 0,99 & 1,02 \\
2007 & 1,20 & 0,91 & 1,01 & 0,94 & 1,11 & 1,14 & 1,27 & 1,02 \\
2008 & 2,03 & 1,14 & 1,13 & 1,81 & 1,22 & 1,29 & 1,83 & 1,02 \\
\hline
\end{tabular}

Fuente: BCE, Casas comerciales, MAG, INE

Tomando los índices de precios de los principales insumos directos y la estructura en la participación en los costos de cada uno de los productos que integran la canasta de bienes de cada una de las provincias, se puede estimar el comportamiento de los insumos en el periodo 2000-2008, tomando como año de base el 2005. El efecto sobre la canasta de productos del grupo objetivo se determinó ponderando el índice de cada producto en relación a su participación promedio en el VBP de la provincia durante el periodo 2003-05. 
Manuel Chiriboga, Edwin Vasquez y Octavio Sotomayor

Cuadro $\mathrm{N}^{\circ} 8$

El Oro: índices de costos directos de producción (Índices 2005-100)

\begin{tabular}{|l|r|r|r|r|}
\hline \multirow{2}{*}{ Años } & Banano & Cacao & \multicolumn{1}{c|}{\begin{tabular}{c} 
Café \\
Indice \\
\cline { 2 - 4 }
\end{tabular}} & \multicolumn{3}{|c|}{ El Oro } \\
\hline 2000 & 71,9 & 45,2 & 57,9 & \\
2001 & 83,0 & 62,9 & 48,2 & 71,3 \\
2002 & 88,8 & 64,4 & 54,4 & 81,8 \\
2003 & 93,7 & 74,1 & 67,6 & 87,9 \\
2004 & 100,0 & 88,0 & 86,9 & 93,4 \\
2005 & 101,5 & 100,0 & 100,0 & 100,0 \\
2006 & 107,6 & 100,6 & 97,7 & 101,4 \\
2008 & 144,2 & 114,5 & 116,6 & 108,0 \\
\hline
\end{tabular}

Fuente: BNF, MAG-SICA, Productores Elaboración: OCE/IICA

En el caso de El Oro el costo de insumos directos de cada uno de los cultivos ha tenido un crecimiento constante durante el periodo de análisis. Los productos que sufrieron el mayor incremento en costos fueron café y cacao, debido a la mayor partici- pación de fertilizantes en sus estructuras de costos. Si bien en banano también hay un crecimiento continuo en costos, la mayor incidencia se presenta en el último año por el fuerte aumento de precio en los fungicidas, rubro de mayor peso en su producción.

Cuadro $\mathrm{N}^{\circ} 9$

Los Ríos: índices de costos directos de producción (Índices 2005-100)

\begin{tabular}{|l|r|r|r|r|r|}
\hline \multirow{2}{*}{ Años } & Arroz & Maíz duro & Cacao & Soya & \multirow{2}{*}{$\begin{array}{c}\text { Índice } \\
\text { Los Ríos }\end{array}$} \\
\cline { 2 - 4 } & \multicolumn{3}{|c|}{ Indice } \\
2000 & & & 45,2 & 68,8 & \\
2002 & 66,5 & 71,1 & 62,9 & 75,4 & 67,4 \\
2003 & 65,4 & 74,1 & 64,4 & 81,6 & 68,4 \\
2004 & 71,6 & 75,7 & 74,1 & 82,2 & 73,8 \\
2005 & 94,0 & 89,8 & 88,0 & 87,3 & 91,3 \\
2006 & 100,0 & 100,0 & 100,0 & 100,0 & 100,0 \\
2007 & 88,9 & 99,3 & 100,6 & 95,5 & 94,3 \\
2008 & 106,6 & 112,9 & 114,5 & 102,6 & 109,5 \\
& 195,8 & 153,4 & 171,2 & 161,8 & 178,3 \\
\hline
\end{tabular}

Fuente: BNF, MAG-SICA, Productores

Elaboración: OCE/IICA 
En la provincia de Los Ríos, el mayor efecto del crecimiento de precios de insumos se da en el cultivo de arroz, aunque en todos sus cultivos principales el aumento de costos en materiales directos ha sido superior al $50 \%$ respecto al 2005. En este grupo de cultivos los insumos de mayor peso son los fertilizantes, cuyos precios registran el mayor crecimiento en el periodo. En el caso de soya, se suma el efecto del fuerte aumento de precios en los fungicidas durante el último año.

Cuadro $\mathrm{N}^{\circ} 10$

Chimborazo: índices de costos directos de producción

(Índices 2005-100)

\begin{tabular}{|l|r|r|r|r|r|}
\hline \multirow{2}{*}{ Años } & Cebada & Papa & Leche & Maíz suave & \multirow{2}{*}{$\begin{array}{c}\text { Índice } \\
\text { Chimborazo }\end{array}$} \\
\cline { 2 - 4 } & \multicolumn{3}{|c|}{ Índice } \\
2000 & 64,8 & & 96,7 & 80,0 & \\
2002 & 58,8 & 54,3 & 60,1 & 83,9 & 59,7 \\
2003 & 58,0 & 67,8 & 71,0 & 82,9 & 70,2 \\
2004 & 65,8 & 78,9 & 106,7 & 78,0 & 96,8 \\
2005 & 87,6 & 91,0 & 95,2 & 83,9 & 93,3 \\
2006 & 100,0 & 100,0 & 100,0 & 100,1 & 100,0 \\
2007 & 97,5 & 98,1 & 102,1 & 101,3 & 100,9 \\
2008 & 120,6 & 11,2 & 114,4 & 115,2 & 113,9 \\
& 191,5 & 182,7 & 146,5 & 162,7 & 158,1 \\
\hline
\end{tabular}

Fuente: BNF, MAG-SICA, Productores

Elaboración: OCE/IICA

En el caso de la provincia del Chimborazo los efectos se diferencian en dos grupos de productos. El primero incluye a los cultivos de maíz suave, cebada y papa, con una alta proporción de fertilizantes dentro de sus estructuras de costos directos, y que por lo tanto, han tenido un crecimiento sustantivo en sus costos en los últimos tres años, afectando, principalmente a la cebada $y$, en menor proporción, al maíz suave y a la papa. En el segundo grupo está la leche, cuyo principal componente de costos es la alimentación del ganado, realizada mediante pastos naturales y alimentos balanceados, que ha tenido un menor aumento porcentual en sus costos directos, aunque de una magnitud importante.

\subsection{Impacto sobre los ingresos agríco- las en las provincias seleccionadas}

Con base en la información sobre la evolución de los precios internos 
de los productos e insumos agropecuarios seleccionados y de sus respectivas estructuras de insumo-producto, es posible estimar el cambio en el valor agregado neto por hectárea generado por las variaciones en dichos precios durante los años recientes (2001-2008) ${ }^{3}$. La estimación de los cambios en el valor agregado neto por hectárea permite disponer de un indicador sobre el cambio en los ingresos de los distintos factores que intervinieron en la producción agropecuaria seleccionada. Ello incluye tanto los salarios a la mano de obra, los honorarios a los servicios, las rentas de la tierra, los intereses financieros, y las utilidades del capital. En el caso de pequeños productores, varios de estos factores se concentran en la unidad doméstica, que es a la vez propietaria u ocupante del predio, proporciona su mano de obra familiar y financia parte de los costos de producción. Los factores de producción "externos" suelen ser los servicios de maquinaria o sanitarios requeridos y los prestamos de avío para la producción.

Para el cálculo de los cambios en el valor agregado neto en las producciones seleccionadas, se ha supuesto que la cantidad de insumos por hectárea permanece constante en todo el periodo, es decir que las relaciones insumo-producto cambian únicamente por modificación en los precios relativos de insumos y productos. Las ponderaciones para agregar los índices de productos a nivel de la provincia corresponden a la participación promedio de las hectáreas cosechadas de cada producto en el periodo 2003-05, respecto a la suma de las hectáreas de los productos seleccionados.

No obstante, hasta este punto el análisis de los efectos sobre los ingresos de los factores de producción involucrados en las actividades seleccionadas no permite identificar si esos cambios los ubicaron en una mejor posición frente al acceso a los alimentos y a otros bienes y servicios de la canasta básica de consumo. Recordemos que las condiciones del mercado internacional si bien influyen favorablemente hacia los productores por las posibilidades de una mejora en los precios de sus productos, dependiendo del grado de trasmisión al mercado doméstico, también los afecta desde la perspectiva de consumidores, ya que las alzas de 
precios trae consigo aumentos en los niveles de inflación y por ende el encarecimiento de la canasta de bienes y servicios de consumo.

Por tanto, es importante determinar cuál ha sido la situación del grupo de productores objetivo ante los cambios en sus ingresos frente al crecimiento en el precio de los bienes de la canasta alimenticia. Para ello, los índices de valor agregado resultantes, que se encuentran calculados a precios "corrientes", se han deflactado por el Índice de Precios al Consumidor (IPC) para ajustar sus valores a los cambios en el poder adquisitivo de la moneda ecuatoriana (dólar) y convertirlos en índices de valor agregado "real".
De los resultados obtenidos se puede apreciar que en dos de las tres provincias se presentan cambios positivos en los ingresos de los productores frente al año de base 2005 . La situación más favorable se presenta en la provincia de Los Ríos en donde los ingresos netos mejoraron para el 2007 en un 23\% y para el 2008 en un $112 \%$, luego de una pérdida presentada en el 2006 de $-16 \%$, se observa que los efectos del mercado internacional tuvo mayores influencias en esta zona dado el tipo de productos involucrados fueron los que experimentaron mayores alzas, como el caso de soya, arroz y maíz duro.

Cuadro $\mathrm{N}^{\circ} 11$

Los Ríos: índices de valor agregado real por hectárea

(Índices 2005-100)

\begin{tabular}{|l|r|r|r|r|r|}
\hline \multirow{2}{*}{ Producto } & Arroz & Maíz amarillo & Cacao & Soya & \multirow{2}{*}{$\begin{array}{c}\text { Total } \\
\text { Los Ríos }\end{array}$} \\
\cline { 2 - 4 } & \multicolumn{3}{|c|}{ Duro } \\
\hline 2001 & 65,3 & 101,7 & 83,6 & 68,2 & 74,5 \\
2002 & 56,0 & 113,5 & 153,0 & 139,5 & 99,6 \\
2003 & 61,6 & 87,3 & 143,6 & 134,7 & 93,3 \\
2004 & 111,2 & 102,3 & 99,9 & 96,0 & 106,8 \\
2005 & 100,0 & 100,0 & 100,0 & 100,0 & 100,0 \\
2006 & 58,0 & 95,8 & 114,4 & 113,6 & 83,8 \\
2007 & 90,2 & 115,3 & 165,5 & 168,1 & 123,2 \\
2008 & 267,5 & 120,3 & 168,0 & 173,5 & 211,7 \\
\hline
\end{tabular}

En segundo lugar está la provincia de El Oro que presentan un comportamiento similar a Los Ríos, una pér- dida del balance neto en el 2006 de $7 \%$ y recuperaciones posteriores en un $29 \%$ en el 2007 y $36 \%$ en el 2008 , 
igualmente en los dos últimos años el cambio positivo es constante. En esta provincia, las hectáreas de cacao son las que han tenido el mayor aumento en rentabilidad, mientras que en las de banano, que es el cultivo más importante, su aumento ha sido menor. El banano constituye el cultivo con mayor área sembrada y los pequeños productores de El Oro dependen casi exclusivamente del banano, por lo que cualquier cambio sustantivo en las condiciones de este producto pondría en riesgo las condiciones económicas y sociales de los productores y sus familias.

\section{Cuadro $\mathrm{N}^{\circ} 12$}

El Oro: índices de valor agregado real por hectárea (Índices 2005-100)

\begin{tabular}{|l|r|r|r|r|}
\hline \multirow{2}{*}{ Producto } & Banano & Cacao & Café & $\begin{array}{c}\text { Total } \\
\text { El Oro }\end{array}$ \\
\cline { 2 - 5 } & \multicolumn{3}{|c|}{} & 80,3 \\
2001 & 110,9 & 79,5 & 26,7 & 97,0 \\
2002 & 106,0 & 145,5 & 19,1 & 89,0 \\
2003 & 93,3 & 137,8 & 18,7 & 79,4 \\
2004 & 88,9 & 98,8 & 37,9 & 100,0 \\
2005 & 100,0 & 100,0 & 100,0 & 92,6 \\
2006 & 72,9 & 113,4 & 101,1 & 129,4 \\
2007 & 112,3 & 164,0 & 115,9 & 135,8 \\
\hline
\end{tabular}

Finalmente, en la provincia del Chimborazo, si bien se presentan cambios positivos en el valor agregado por hectárea de cebada y maíz dulce, en los principales productos de la zona se registran reducciones en los respectivos valores agregados, en especial en la producción de leche. Por lo anterior, la situación de ingresos por hectárea en las principales actividades agropecuarias en esta provincia resulta en promedio similar a la existente en el año base e inferiores a la que regía al principio de la década. Este comportamiento difiere al encontrado en las provincias de El Oro y Los Ríos debido a que en esta zona el comportamiento agrícola tiene una baja interrelación con los cambios en los mercados internacionales, predominan estructuras sobre las cuales la trasmisión de precios es más lenta, existe una numerosa red de intermediarios, parte de la producción se destina al autoconsumo y la ausencia de cierto tipo de infraestructura como en el caso de la leche influye en la forma de venta de la producción. 
Precios agropecuarios e ingresos: el caso de tres provincias en Ecuador

Cuadro $\mathrm{N}^{\circ} 13$

Chimborazo: índices de valor agregado real por hectárea

(Índices 2005-100)

\begin{tabular}{|c|c|c|c|c|c|}
\hline \multirow[t]{2}{*}{ Producto } & Рapa & Cebada & Maíz dulce & Leche & \multirow{2}{*}{$\begin{array}{c}\text { Total } \\
\text { Chimborazo }\end{array}$} \\
\hline & & & & & \\
\hline 2001 & 68,6 & 170,1 & 176,0 & 181,8 & 150,8 \\
\hline 2002 & 103,1 & 134,0 & 148,0 & 146,8 & 133,2 \\
\hline 2003 & 74,4 & 94,5 & 87,7 & 87,7 & 86,7 \\
\hline 2004 & 48,2 & 111,7 & 73,5 & 96,7 & 84,3 \\
\hline 2005 & 100,0 & 100,0 & 100,0 & 100,0 & 100,0 \\
\hline 2006 & 103,4 & 94,7 & 111,3 & 93,3 & 100,5 \\
\hline 2007 & 56,2 & 132,3 & 125,5 & 77,8 & 101,1 \\
\hline 2008 & 99,3 & 120,2 & 124,1 & 43,1 & 99,3 \\
\hline
\end{tabular}

En resumen, en el periodo de análisis, en especial los años posteriores al 2005, el balance neto de los ingresos de los pequeños productores de las provincias de El Oro y Los Ríos tuvieron una mejoría, resultado de los efectos de los cambios en el mercado internacional de bienes agrícolas, por el efecto de productos como arroz, maíz y banano. En la provincia del Chimborazo, los mayores ingresos por hectárea en los cultivos de cebada y maíz dulce, se vieron contrarrestados por la caída en la rentabilidad de la producción de leche, y en mucho menor grado por el cultivo de papa, productos con una baja articulación a los cambios en las condiciones externas.

\subsection{Evolución de la producción de los principales productos seleccionados}

Los aumentos de precios agrícolas, si bien han tenido un efecto muy positivo sobre los ingresos por hectárea en varios de los cultivos seleccionados, su efecto sobre el nivel de la producción no es tan notorio en la mayoría de los casos analizados. Esta situación es claramente explicable por la baja elasticidad de oferta que normalmente tienen las producciones agropecuarias, en especial los cultivos permanentes y la actividad pecuaria. En muchos casos, las expectativas de buenos precios por parte de los agricultores los incentivan al uso de mayores y mejores insumos para asegurar mayores ren- 
dimientos por hectárea. Sin embargo, el abrupto crecimiento en los precios de algunos insumos, principalmente los derivados del petróleo, pueden haber desanimado una mayor inversión en los cultivos.

En el caso del banano, el comportamiento de la producción en los años posteriores al 2005 fue muy positivo y estuvo determinado en mayor medida por un crecimiento moderado de los rendimientos y por la recuperación del área cosechada que se tenía a inicios de la década. El número de hectáreas cosechadas ha fluctuado relativamente poco durante el periodo, respecto al promedio 2003-05, con un rango 10\% arriba y debajo de dicho promedio. El área cultivada de banano tiene relación directa con varios factores como son la política interna de precios oficiales de la caja de exportación, el comportamiento de los mercados internacionales, en especial las cotizaciones de la fruta, las políticas internas de control de nuevas siembras de banano y las condiciones climáticas en la zona que influye directamente en la cosecha del producto.

En el caso del cacao, las tendencias de la superficie cultivada y los rendimientos en el periodo 20002008 son decrecientes, tanto en la provincia de El Oro como en la de Los Ríos. A pesar del continuo incre- mento de la rentabilidad del cacao desde el 2004, el comportamiento del área cultivada y de los rendimientos ha sido decreciente. El comportamiento de los años previos al alza de precios que presentó situaciones variables, pudo haber sido un determinante para que algunos pequeños productores busquen otras alternativas de cultivo con mejores perspectivas, entre ellas el banano, maíz duro, el mismo arroz y otros productos como palma africana y maracuyá. El cacao es un producto que requiere de cierto tiempo para observar el efecto de determinados factores sobre las expectativas de los productores, en ese sentido si los precios altos de los últimos años incentivan nuevas siembras, sus resultados recién se apreciarán en el mediano plazo, dependiendo de la evolución de las condiciones en los productos alternativos.

En café, la superficie cosechada ha marcado una tendencia estable en el periodo 2000-2004 y con un descenso entre el 2006 y 2008, sin embargo, los niveles de rendimiento presentan una mejora sustantiva para los años 2007 y 2008. La reacción de los productores de café es diferente a los del cacao frente al comportamiento de los precios, ya que los rendimientos mantienen una tendencia muy similar a los precios durante el periodo 
de análisis. En cuanto a los pequeños productores de café sus prácticas de cosecha están en función de la situación del mercado, a épocas de precios buenos cosechan más café sin necesidad de aumentar la superficie existente o modificar sus patrones de cultivo y viceversa. Los efectos: precios y rendimiento han permitido que los ingresos brutos de los productores hayan mejorado en los años 2007 y 2008 en niveles mayores a los de banano y cacao inclusive.

Los altos precios del arroz, registrados tanto en el mercado internacional como en el mercado interno en el 2008, no lograron promover una mayor cosecha de esta gramínea. La fuerte caída en el área cosechada en la provincia de Los Ríos, motivada por la adversa situación climática que se presentó en el primer trimestre del 2008, ocasionó una significativa disminución en la producción. Lo anterior se dio a pesar de la mejora en los rendimientos que ha ocurrido desde el 2005, y que fue reforzada por programas gubernamentales de ayuda mediante entrega de paquetes agropecuarios (semillas certificadas, urea, agroquímicos) a los pequeños agricultores afectados.

Se puede afirmar que el cultivo del arroz ha logrado mejoras en cuanto a sus niveles de productividad. En varias zonas, donde hay pre- sencia de pequeños productores, se han realizado importantes obras de infraestructura, en especial de riego, que les ha permitido incrementar sus niveles productivos y poder contar con producciones en dos épocas del año, equilibrando de alguna manera los picos de producción para evitar sobreproducción excesiva que afecte los niveles de precios internos. Estos cambios en el sector se han presentado en los últimos cinco años logrando una importante estabilidad y favorables perspectivas para los productores. A esto se suman las políticas públicas basadas en un mecanismo arancelario de estabilización de precios como es el Sistema Andino de Franja de Precios y medidas de protección en frontera para evitar el ingreso de arroz importado. Estos aspectos de política han influenciado el comportamiento de los precios durante el periodo 2000-2008, al observarse una tendencia creciente en relación al periodo de base.

El maíz duro $\mathrm{O}_{2}$ otro de los cultivos de importancia en el grupo de pequeños productores, la tendencia del área cultivada y de los rendimientos ha sido muy positiva a partir del 2005. La producción en el 2008 más que duplica el nivel promedio del periodo base (2003-05). El cultivo del maíz se caracteriza por su vinculación al sector agroindustrial, en 
especial la carne de pollo, cerdo y camarón, ya que constituye un componente del alimento balanceado junto con la torta de soya. La dinámica de estos sectores de la cadena alimenticia y exportadora del Ecuador influye en el comportamiento del cultivo del maíz duro.

Finalmente los cultivos de soya, que también fueron afectados por el clima adverso a fines del 2007 e inicios del 2008, han registrado menores áreas y más bajos rendimientos que en los primeros años de la déca$\mathrm{da}$, a pesar de la importante mejora en los precios internos a partir del 2005.

La producción de leche, uno de los principales rubros para la economía agraria en Chimborazo, se mantuvo relativamente estable hasta el año 2005, pero se incrementó en los años 2006, 2007 y 2008. Estos cambios se presentaron por un mejoramiento en los niveles de rendimiento en algunos casos y en otros por un cambio hacia la producción de leche de algunos productores de hortalizas en la zona. A nivel de rendimientos se observó una tendencia moderadamente creciente, salvo el incremento registrado en el 2006, año en el cual hay un mejoramiento en las condiciones productivas.

La cebada ha tenido un fuerte aumento en producción en los últi- mos tres años, motivado tanto por una mayor área cosechada como por un aumento en los rendimientos por hectárea. Esta mejora en rendimientos $(27 \%$ superior al periodo base 2003-05) de los productores de cebada en Chimborazo debe considerarse un aumento en rentabilidad por encima de lo obtenido a causa del aumento de precios internos, que fue de $32 \%$ por hectárea en el 2007 y de $20 \%$ en el 2008 bajo el supuesto de rendimientos constantes similares al promedio del periodo base. Es claro que este grupo de productores recibieron de alguna forma también los efectos del incremento de las cotizaciones internacionales de este producto.

En el caso del cultivo de papa, de gran importancia en la canasta de los pequeños agricultores, su producción está influenciada directamente por factores internos, pues es un producto poco comercializado a nivel internacional. De la evolución de los índices se puede apreciar que existe una relación directa entre las fluctuaciones de las hectáreas y el precio de la papa, a incrementos de la superficie se presentan reducciones de precios y viceversa, lo cual es característico de este producto. La producción de papa se consume en una alta proporción en fresco; según estudios de este cultivo más del $90 \%$ de la pro- 
ducción se comercializa en mercados mayoristas y restaurantes, un porcentaje pequeño se lo destina a la agroindustria, en especial para la producción de "papas chips". Esta estructura en la comercialización hace que este cultivo tenga un comportamiento muy variable y los productores se comporten de acuerdo a las condiciones del mercado. Más que una influencia de precios internacionales, la papa es afectada por ciclos productivos y políticas de intermediarios en el mercado interno.

La producción de maíz suave en la provincia del Chimborazo ha estado descendiendo continuamente desde inicios de la presente década, aunque luego del 2005 se nota alguna recuperación de áreas de cultivo pero con un rendimiento relativamente bajo respecto al rendimiento histórico.

En resumen, de los diez productos estudiados, que corresponden a las producciones más importantes en las provincias seleccionadas, cuatro de ellos (maíz amarillo duro, cebada, café y banano) han respondido con aumentos importantes en su producción frente a los aumentos de precios internos, vinculados con la evolución de los precios internacionales. En maíz amarillo duro y cebada, por ser cultivos de ciclo corto, la respuesta de los productores ha sido la de incrementar áreas y también mejorar la aplicación de insumos modernos que han repercutido en aumento de rendimientos. En los productos de exportación (café y banano) los aumentos han venido por incrementos en rendimientos, pues los aumentos en área recién tienen su impacto luego de varios años.

Otros tres cultivos, claramente vinculados al mercado internacional como el arroz, cacao y soya, y cuyos precios internos han aumentado fuertemente en el 2008, no han respondido con aumentos de producción en las respectivas provincias. En algunos de estos casos, la razón es de carácter climático, pues dichos cultivos fueron afectados por inundaciones a inicios del 2008.

Finalmente, en los casos de las producciones de papa y leche, sus niveles de producción están más afectados por consideraciones del mercado interno, $y$ sus precios internos han variado independientemente de las tendencias internacionales de productos similares.

\section{Políticas públicas aplicadas ante la coyuntura del aumento de precios y el acceso de los productores}

La economía mundial hasta mediados del 2008 comenzó a experimentar un proceso de repunte 
inflacionario, resultado fundamentalmente del inusitado incremento de precios de materias primas como petróleo, gas, minerales y alimentos. Esta situación conllevó a que instituciones vinculadas al desarrollo y la alimentación como la FAO, el Banco Mundial, entre otros, comiencen a presentar sus preocupaciones sobre el futuro de la alimentación mundial, es más, las diferentes variables mundiales relacionadas a la seguridad alimentaria, avizoraban posibles problemas de abastecimiento de alimentos en países en desarrollo y menos adelantados.

Los repuntes inflacionarios comenzaron a golpear a todas las economías, en especial a las en desarrollo; en agosto del 2008 el promedio de la inflación global llegó al 8\% y las economías en desarrollo al 6\% en promedio, sin embargo se presentaron niveles de dos dígitos en algunos países como Venezuela (30\%), Argentina (10,5\%), Rusia (15\%), entre otros.

Este problema no fue diferente para el Ecuador, el nivel de inflación que para el año 2007 fue del 3\% se incrementó hasta agosto del 2008 al $10 \%$, influenciado mayormente por el incremento de precios en los alimentos que pasó del 5\% en el 2007, al $23 \%$ en el 2008. El peso de los productos alimenticios de origen impor- tado tuvieron un impacto en la inflación de los alimentos en un $11 \%$, básicamente por productos como trigo, maíz, cebada, soya, entre otros.

Entre los factores que influyeron en este crecimiento inflacionario estaba el mayor precio del componente importado de los alimentos, el incremento en los costos de producción agrícola, fundamentalmente, en los insumos que en un gran porcentaje es importado, el aumento de los flujos de remesas los cuales se dirigieron a la adquisición de bienes de consumo y una política fiscal expansionista que aumento la demanda interna por bienes, en especial importado.

Bajo este escenario, el Gobierno Nacional se vio en la necesidad de implantar un conjunto de políticas hacia dos objetivos: reducir la escala de precios y los costos de producción de los bienes agrícolas.

Entre las políticas que se implementaron durante el 2008 estuvieron las siguientes:

Medidas orientadas a mitigar efectos inflacionarios

- Fijación de precios de la leche al productor y al consumidor.

- Eliminación de aranceles para las importaciones de trigo, sémola, tranquillón. 
- Prohibición de exportación de arroz total excepto a Venezuela y fijación de precios piso $y$ techo del arroz.

- Subsidio directo a la harina argentina importada y al resto de harinas importadas o producidas en el País.

- Estabilización y fijación de precios máximos de productos de la canasta básica alimenticia.

- Programa socio-solidario.

- Programa socio-tienda.

Medidas orientadas a reducir los costos de producción

- Subsidio para los insumos agroquímicos agrícolas y programa socio-siembra.
- Venta de urea a precio subsidiado.

- Exoneración del IVA a todos los insumos y bienes de capital agrícolas.

- Eliminación del pago del Impuesto a la Renta para importadores de agroquímicos $y$ productores agrícolas.

- Unificación y reducción de las tarifas eléctricas.

A pesar de las políticas implementadas, el acceso de los productores en las tres provincias investigadas al parecer fue reducido, de acuerdo a respuestas proporcionadas por los mismos productores. En el siguiente gráfico se aprecia los niveles de respuesta presentadas en las tres provincias en función del tipo de medida de política a la que tuvieron acceso.

Cuadro $N^{\circ} 14$

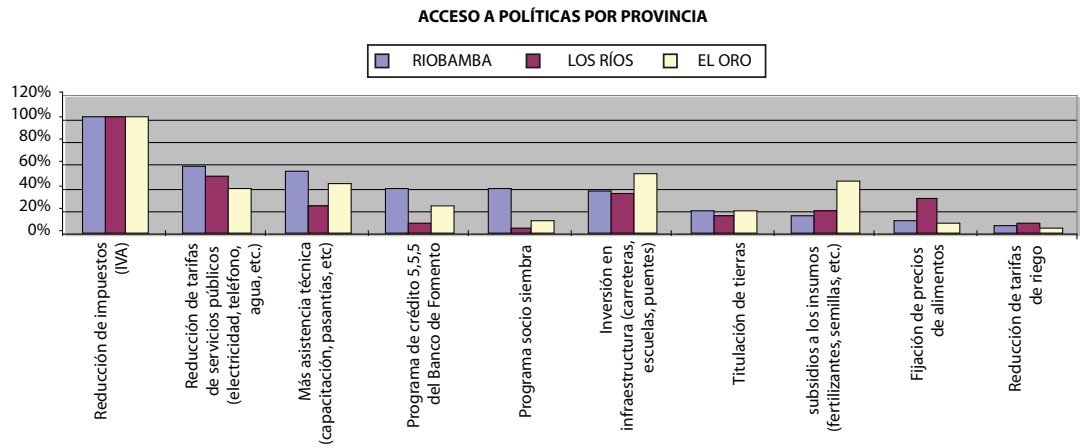

Fuente: Encuesta

Elaboración: OCE 
En resumen, si bien el Gobierno implementó un conjunto de políticas orientadas a favorecer a los pequeños productores, éstas tuvieron baja cobertura. El número de productores entrevistados no se efectuó sobre la base de una muestra representativa, para poder extrapolar los resultados al conjunto de productores, si llama la atención que entre los entrevistados la mayoría de ellos no tuvieron acceso a las políticas puestas en marcha por el gobierno, salvo aquellas de mayor facilidad en su operatividad como reducción de IVA.

En ese sentido, es importante realizar una investigación más amplia sobre los beneficios reales de dichas políticas y el costo en que ha incurrido el Gobierno para su ejecución, más aún cuando algunas de ellas se las ejecutaron sin una adecuada planificación previa e información clara de los beneficiarios.

\section{Conclusiones}

De los resultados obtenidos en el presente estudio, que tuvo como objetivo medir el impacto que sobre los productores agrarios generó el incremento de precios en los productos básicos, se pueden extraer las siguientes conclusiones.

1. El Ecuador se caracteriza por tener un importante nivel de autosu- ficiencia alimentaria, soportada en un alto porcentaje por su producción interna en varios de los principales grupos de alimentos de consumo de su población. Sin embargo, las importaciones de alimentos en los últimos ocho años presentan un comportamiento ascendente, principalmente en algunos productos como aceites vegetales y cereales.

2. En relación al nivel de vulnerabilidad de la economía ante cambios en los mercados agrícolas, el Ecuador en los últimos años ha presentado una posición favorable para hacer frente a esta coyuntura, dado por la evolución positiva del PIB y en especial del INB percápita que reflejó la ganancias obtenidas en los términos de intercambio externos. Las fuentes principales de financiamiento de la economía y de la provisión de alimentos importados fueron fundamentalmente los ingresos provenientes de las exportaciones de bienes y las remesas originarias de los inmigrantes, que hasta mediados del 2008 mantuvieron un comportamiento positivo.

3. En todos los productos se observó que en el periodo 20062008, experimentaron en diferentes magnitudes crecimientos en los precios al productor. Este comportamiento permitió que exista mejoras en el nivel de los ingresos brutos en 
cada uno de los productos, y en el conjunto de los productores por provincia la magnitud de esos cambios positivos estuvo influenciada por el producto o productos de mayores pesos en el ingreso bruto de la zona. Sin embargo, este comportamiento positivo hubiera sido más favorable si se hubieran presentado mejoras en los niveles de producción para el conjunto de productos, si bien algunos de ellos reaccionaron positivamente, ya sea mediante mejoras de rendimiento o incremento de superficie, el balance global no fue muy dinámico.

4. En relación a los costos de los principales insumos directos de producción también sufrieron importantes incrementos durante el periodo de análisis, concentrándose básicamente en fertilizantes que constituye el rubro de mayor peso en los costos de producción de la mayoría de cultivos, seguido por los fungicidas que tienen una alta participación para el caso del banano y en menor proporción el alimento balanceado en relación a la leche. La provincia que tuvo los mayores impactos en costos fue Los Ríos, seguida del Chimborazo y después El Oro, este último por el peso del banano producto cuya participación de los fertilizantes es menor, pero con un alto peso en el uso de pesticidas.
5. En el balance neto los ingresos de los pequeños productores de las provincias de Los Ríos y El Oro tuvieron una mejoría, resultado de los efectos de los cambios en el mercado internacional de bienes agrícolas, presentándose crecimientos en el índice del valor agregado para todos los productos. Por su parte en la provincia del Chimborazo, el resultado del conjunto de productores mantuvo las condiciones existentes en el 2005, caracterizado por mayores ingresos por hectárea en los cultivos de cebada y maíz dulce, viéndose contrarrestados por la caída en la rentabilidad de la producción de leche, y en mucho menor grado por el cultivo de papa, productos con una baja articulación a los cambios en las condiciones externas.

6. A nivel de producto los mayores niveles de rentabilidad se presentaron en aquellos que reflejan una mayor trasmisión de los precios internacionales hacia el mercado doméstico, en algunos casos con menores intervenciones del Estado y articulados de alguna forma al mercado de exportación, productos como: el cacao, el café, el arroz y en menor medida el banano, y en otros casos con un integración hacia la agroindustria como son el caso del maíz duro y la soya. 


\section{Manuel Chiriboga, Edwin Vasquez y Octavio Sotomayor}

7. El Gobierno implementó un conjunto de políticas orientadas a favorecer a los pequeños productores, éstas no lograron cubrir a todo el conjunto de productores, tuvieron mayor acceso en aquellas políticas de mayor facilidad en su operatividad como fueron la reducción de IVA, tarifas de servicios públicos y asistencia técnica, no así aquellas que requieren de mayor logística o difusión para su cobertura. 